\title{
IMPACTO DA INFORMAÇÃO SOBRE A MELATONINA NA ACEITABILIDADE DE LEITE
}

\author{
MARIA PATRÍCIA MILAGRES * \\ VALÉRIA PAULA RODRIGUES MINIM ** \\ ANDRÉA ALVES SIMIQUELI *** \\ LUÍS ANTÔNIO MINIM **** \\ RAFAEL SCORALIK GOLDNER ***** \\ BETANIA FERNANDES GALVÃO ******
}

\begin{abstract}
O objetivo deste trabalho foi avaliar a influência da informação e do conhecimento sobre a melatonina na aceitação de leite. Foram recrutados 200 voluntários (idade inferior e superior a 50 anos) para avaliarem o leite de vaca pasteurizado com concentração aumentada de melatonina mediante teste cego, teste da informação da presença de melatonina e teste do conhecimento sobre melatonina. As sessões foram realizadas em condições laboratoriais, utilizandose escala hedônica de 7 pontos, sendo os resultados analisados por ANOVA e teste t. O leite com informação da presença de melatonina alcançou maior escore quando comparado ao teste cego em ambos os grupos. A comparação dos resultados dos testes sem e com conhecimento sobre a melatonina indicou que a noção dos benefícios do composto favoreceu a aceitação do produto, principalmente no grupo com idade superior a 50 anos. Conclui-se que os consumidores estão preocupados com a saúde e dispostos a aceitar produtos com características funcionais.
\end{abstract}

PALAVRAS-CHAVE: MELATONINA; ACEITABILIDADE E INFLUÊNCIA DA INFORMAÇÃO.

\footnotetext{
* $\quad$ Professora, Doutora em Ciência e Tecnologia de Alimentos, Universidade Estadual do Sudoeste da Bahia (UESB), Jequié, BA (e-mail: patricia@uesb.edu.br).

** Professora, Doutora em Ciência de Alimentos, Universidade Federal de Viçosa (UFV), Viçosa, MG (e-mail: vprm@ufv.br).

*** Mestranda em Ciência e Tecnologia de Alimentos, UFV, Viçosa, MG (e-mail: andrea.simiqueli@ufv.br).

**** Professor. Doutor em Engenharia Química, UFV, Viçosa, MG (e-mail: Iminim@ufv.br).

***** Graduando em Agronomia, UFV, Viçosa, MG (e-mail: skolra@hotmail.com).

****** Graduanda em Ciências e Tecnologia de Laticínios, UFV, Viçosa MG (e-mail: betaniafernandesg @hotmail.com).
} 


\section{INTRODUÇÃO}

A análise sensorial tradicional, que enfoca os atributos intrínsecos ao produto, tem sido utilizada para estudar as características sensoriais provenientes do equilíbrio de compostos presentes nos alimentos a fim de garantir seu padrão de qualidade. Para atingir a qualidade exigida pelo consumidor, o alimento precisa de respaldo no que diz respeito à sua aparência, aroma, sabor e textura. Entretanto, as ferramentas tradicionais da análise sensorial não são suficientes para atender aos requerimentos do dinâmico mercado de produtos alimentícios. Otimizar a formulação do produto é necessário para sua aceitação e para garantir que a inovação alcance sucesso. Os consumidores, porém, são influenciados por outros fatores durante o processo de escolha, compra ou aceitação do alimento, como conceitos individuais (aprendidos, vivenciados ou instintivos), além de sofrerem influência das informações relacionadas ao produto, como marca, preço e rótulo. Esses fatores, ou características não sensoriais, são veiculados na embalagem do alimento e envolvem os conceitos do próprio consumidor em relação ao produto (MINIM, 2010).

Trabalhos anteriores (VIDIGAL et al., 2011; DELLA LUCIA et al., 2010; JAEGER, 2006; ALLISON, GUALTIERI e CRAIG-PETSINGER, 2004; PRESCOTT et al., 2002) divulgaram a influência de características extrínsecas ao alimento na percepção do seu sabor, revelando como outras informações, além das suas características físicas e sensoriais podem modificar a escolha e a aceitação do consumidor.

Entre os fatores não sensoriais que afetam o comportamento dos consumidores destacamse aqueles relacionados à saúde e ao bem-estar fisico e mental (ALLISON, GUALTIERI e CRAIGPETSINGER, 2004). Informações relevantes sobre o conteúdo nutricional e benefícios à saúde estão sendo amplamente utilizadas como estratégia de marketing e diferenciação do produto, devido ao seu potencial para influenciar as atitudes dos consumidores (SABBE et al., 2009; HAILU et al., 2009; SIEGRIST, STAMPFLI e KASTENKOLZ, 2008). O potencial dessas alegações depende de vários fatores, incluindo os benefícios e familiaridade com os ingredientes (VAN TRIJP e VAN DER LANS, 2007; VAN KLEEF, VAN TRIJP e LUNING, 2005), bem como características individuais dos consumidores, tais como atitudes, motivação e condições de saúde (URALA e LÄHTEENMÄKI, 2004; VAN KLEEF, VAN TRIJP e LUNING, 2005).

Sabbe et al. (2009) estudaram a influência da informação sobre os benefícios à saúde do suco de açaí na aceitação dos consumidores. Usaram concentrações de $4 \%$ a $20 \%$ de polpa e observaram correlação negativa entre a aceitabilidade global do suco e a concentração de açaí. No entanto, verificaram melhora na aceitação dos sucos ao fornecerem informações aos julgadores sobre seus benefícios à saúde, além de redução da insatisfação com o sabor dos produtos.

Diversos alimentos com propriedades funcionais têm sido desenvolvidos empregando a melatonina, composto produzido pela glândula pineal com importante função na regulação do sono dos mamíferos (KENNAWAY e WRIGHT, 2002). O desenvolvimento de leite com concentração de melatonina aumentada naturalmente por meio de técnicas de ordenha criou segmento rentável no mercado de lácteos. De acordo com Özer e Kirmaci (2010), uma empresa na Finlândia produz leite com maior concentração de melatonina usando o sistema de ordenha noturna. Produtos similares são encontrados no Japão e no Reino Unido, porém não foram localizadas pesquisas a respeito da opinião dos consumidores sobre esse produto.

O estudo com consumidores assume importância para definir a aceitação de novos produtos e sua opinião passou a ser referida como o principal fator de sucesso para os alimentos funcionais. Como consequência, várias pesquisas têm enfocado especificamente as atitudes dos consumidores em relação aos alimentos funcionais, abordando determinantes cognitivos, motivacionais e atitudes de aceitação (BECH-LARSEN e GRUNERT, 2003; URALA e LÄHTEENMÄKI, 2004; VERBEKE, 2005).

O presente trabalho teve como objetivo avaliar a influência da informação e do conhecimento sobre a presença de melatonina na aceitação de leite. 


\section{MATERIAL E MÉTODOS}

Iniciou-se o trabalho após sua aprovação pelo Comitê de Ética em Pesquisa com Seres Humanos da Universidade Federal de Viçosa (UFV), sob o protocolo n. 021/2010.

Utilizou-se leite de vaca integral com concentração aumentada de melatonina, obtido em ordenhas noturnas de animais do estábulo leiteiro da UFV, e pasteurizado no Laticínio Escola da referida Universidade.

Duzentos voluntários residentes na cidade de Viçosa (MG) foram recrutados e divididos em dois grupos, sendo o primeiro constituído por 100 pessoas com idade inferior a 50 anos e o outro por 100 pessoas com idade superior a 50 anos.

Os testes de aceitação foram conduzidos em cabines individuais, sob luz branca, divididos em três sessões. Inicialmente realizou-se o teste cego, em seguida o teste da informação e, posteriormente, o teste do conhecimento (Figura 1).

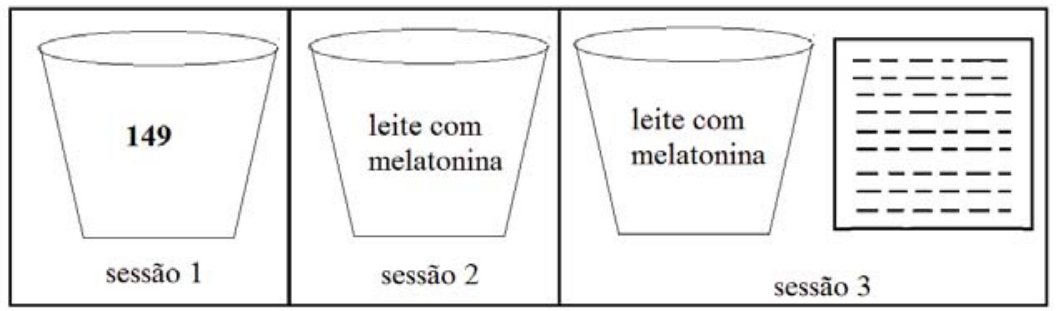

\section{FIGURA 1 - PROCEDIMENTO DO EXPERIMENTO DE INFLUÊNCIA DE FATORES NÃO SENSORIAIS}

Na primeira sessão (teste cego), os consumidores degustaram a amostra do produto servida em copo de acrílico (capacidade de $40 \mathrm{~mL}$ ), codificada com 3 dígitos, sem qualquer informação prévia sobre o leite testado. Na segunda sessão (teste da informação), a mesma amostra de leite (da sessão 1) foi servida aos voluntários, sendo informado que o leite continha melatonina. Na terceira sessão (teste do conhecimento) avaliou-se a importância do conhecimento sobre a melatonina na aceitação do produto. A mesma amostra da sessão 2 foi servida aos voluntários, sendo fornecido texto informativo antes da sessão (Figura 2).

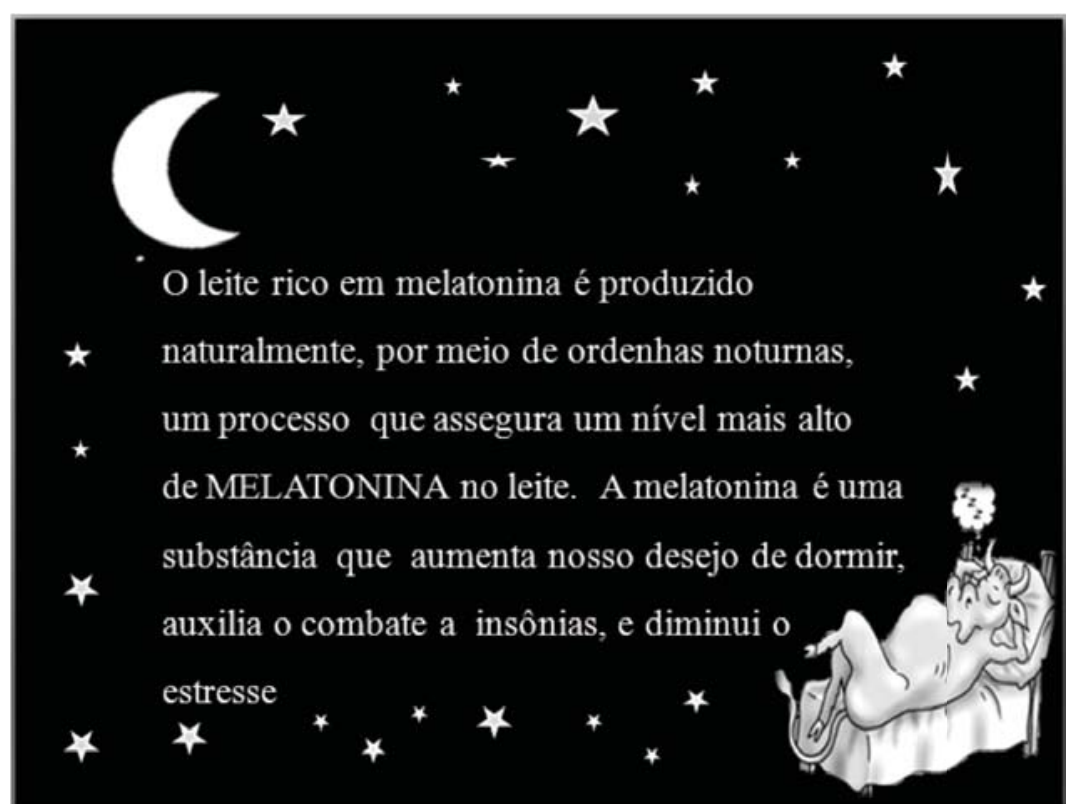

FIGURA 2 - INFORMAÇÃO SOBRE A MELATONINA FORNECIDA NA SESSÃO 3 DO TESTE 
As amostras de leite, das três sessões, foram servidas de forma monádica. Em todas as sessões, o julgador recebeu uma ficha para cada amostra, Figura 3, em que foi solicitado indicar em escala hedônica de 7 pontos sua aceitação do produto (MINIM, 2010).

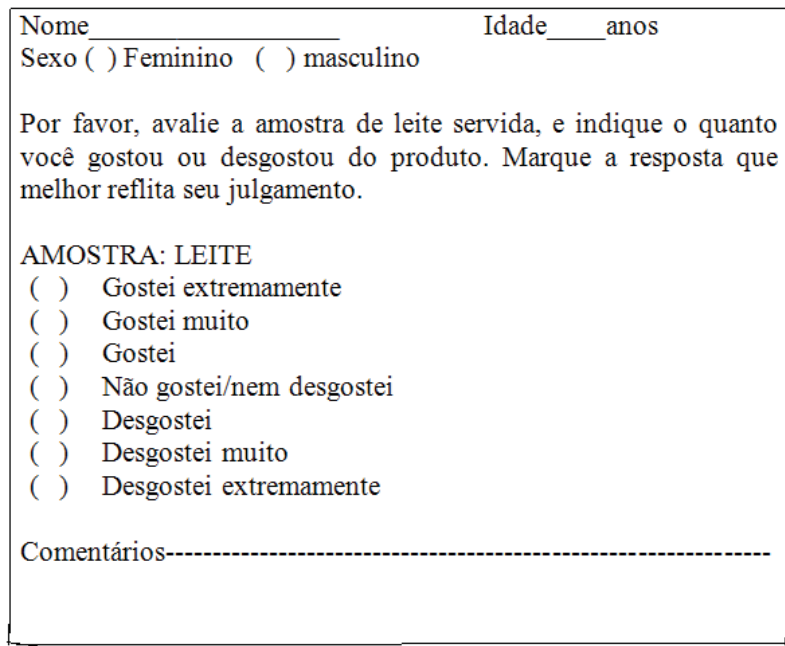

(a)

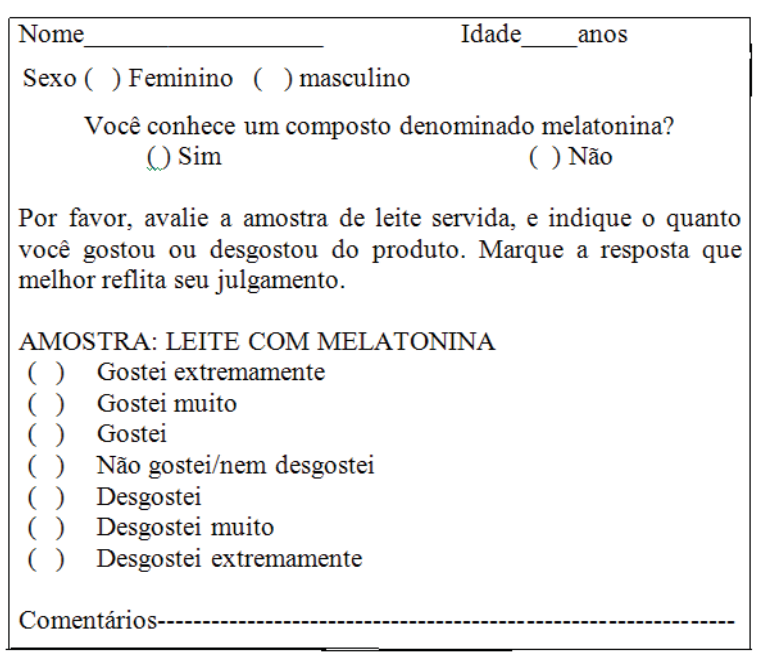

(b)

\begin{tabular}{|l|}
\hline Nome_ \\
Sexo ( ) Feminino ( ) masculino \\
Por favor, avalie a amostra de leite servida, e indique o quanto \\
você gostou ou desgostou do produto. Marque a resposta que \\
melhor reflita seu julgamento. \\
AMOSTRA: LEITE COM MELATONINA \\
( ) Gostei extremamente \\
( ) Gostei muito \\
( ) Gostei \\
( ) Não gostei/nem desgostei \\
( ) Desgostei \\
( ) Desgostei muito \\
( ) Desgostei extremamente \\
Comentários--------------------------------------------------
\end{tabular}

(c)

FIGURA 3 - FICHAS DE AVALIAÇÃO SENSORIAL USADAS: (a) SESSÃO 1; (b) SESSÃO 2; (c) SESSÃO 3 
Para facilitar o entendimento dos resultados efetuou-se a análise de frequência dos escores hedônicos para cada amostra e sessão, conforme as seguintes faixas:

Faixa 1 = escores de 5 a 7 - situados entre os termos hedônicos gostei extremamente e gostei, indicando que os consumidores gostaram da amostra.

Faixa 2 = escores de 1 a 4 - situados entre os termos hedônicos não gostei/nem desgostei e desgostei extremamente, indicando que os consumidores não gostaram da amostra.

Realizou-se análise de variância $(p<0,05)$ para comparação entre as três sessões e também entre os dois grupos. $O$ teste $t$ para amostras pareadas $(p<0,05)$ foi utilizado para comparação entres as sessões 1 e 2, 2 e 3 .

As análises estatísticas foram efetuadas usando-se o programa Statistical Analysis System (SAS, 2008), versão 9.1, licenciado para a Universidade Federal de Viçosa.

\section{RESULTADOS E DISCUSSÃO}

Pelo resultado da análise de frequência pode-se observar aumento do percentual de escores positivos quando foi fornecida a informação da presença de melatonina e também ao serem apresentados os benefícios do seu consumo (Figura 4).
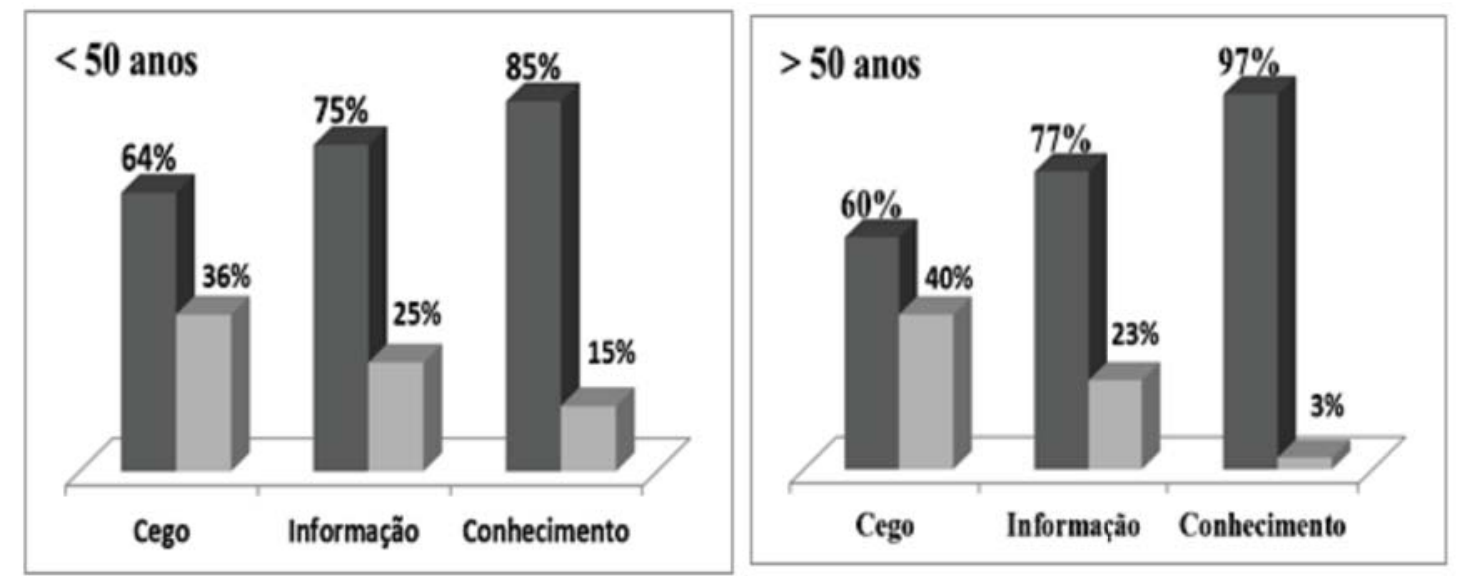

\section{FIGURA 4 - FREQUÊNCIA DE NOTAS HEDÔNICAS \& POSITIVAS (ESCORES DE 5-7) E NEGATIVAS (ESCORES DE 1-4) NAS TRÊS SESSÕES}

Constatou-se diferença significativa entre as médias de aceitação dos leites avaliados nas três sessões, conforme o teste de Tukey $(p<0,05)$, mostrando a influência positiva da informação da presença de melatonina e do conhecimento sobre os benefícios do consumo do leite com concentração aumentada de melatonina.

Também foi verificada diferença nos escores de aceitação de ambos os grupos: com idade superior e inferior a 50 anos (Figura 5).

\subsection{COMPARAÇÃO ENTRE AS SESSÕES}

O teste $t$ para amostras pareadas detectou diferenças significativas $(p<0,05)$ entre os escores obtidos para a aceitação do leite entre as sessões 1 e 2 (Tabela 1). O leite alcançou escore maior no teste com informação da presença de melatonina quando comparado ao teste cego em ambos os grupos (idade superior e inferior a 50 anos). Pode-se observar que a neofobia alimentar não influenciou a aceitação dos consumidores, pois a maioria (82 \%) relatou que desconhecia o composto denominado melatonina na ficha de análise sensorial (Figura 3 b). Mesmo assim, os escores de aceitação aumentaram quando foi fornecida a informação sobre a presença de melatonina no leite. 


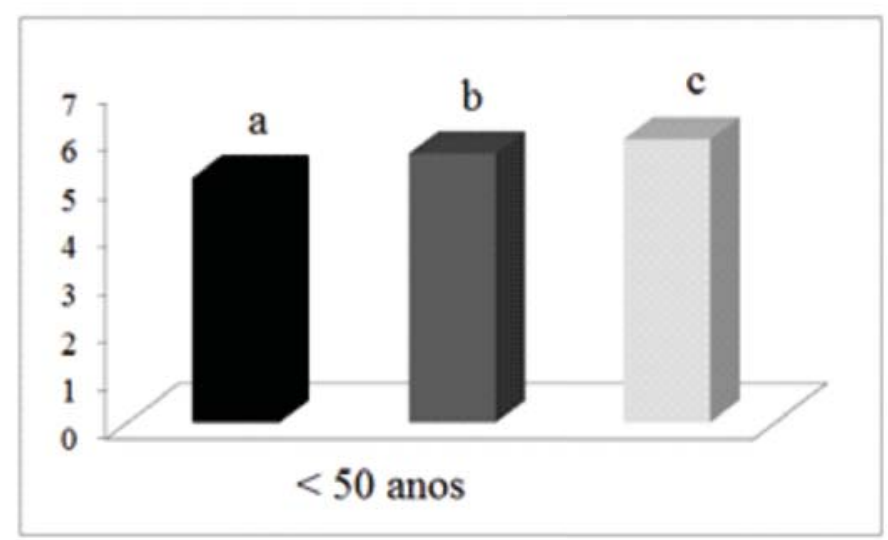

(a)

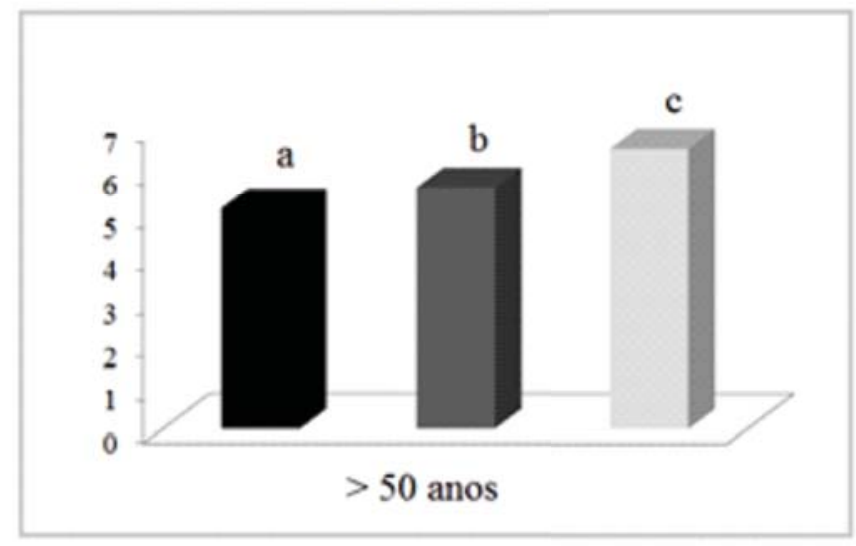

(b)

FIGURA 5 - MÉDIAS DOS ESCORES DE ACEITAÇÃO DO LEITE COM CONCENTRAÇÃO AUMENTADA DE MELATONINA AVALIADO PELO - TESTE CEGO, - TESTE COM INFORMAÇÃO SOBRE A PRESENÇA DE MELATONINAE — TESTE COM CONHECIMENTO SOBRE OS BENEFÍCIOS DO CONSUMO DE MELATONINA PELOS GRUPOS DE MENORES DE 50 ANOS (a) MAIORES DE 50 ANOS (b)

Médias com a mesma letra não diferem significativamente pelo teste tukey $(p<0,05)$.

TABELA 1 - COMPARAÇÃO ENTRE AS SESSÕES 1 E 2 USANDO TESTE T PARA AMOSTRAS PAREADAS

\begin{tabular}{lccc}
\hline \hline \multirow{2}{*}{$\begin{array}{l}\text { Voluntários } \\
\text { Menores de } 50 \text { anos }\end{array}$} & Sessão 1 & Sessão 2 & $\rho$-valor* \\
\cline { 2 - 4 } Maiores de 50 anos & 5,1 & 5,6 & $<0,0001$ \\
\hline \hline
\end{tabular}

${ }^{*} \rho$-valor obtido no teste t para amostras pareadas. 
Define-se a neofobia alimentar como a resistência ao consumo de alimentos desconhecidos, que pode ser responsável pelo insucesso de produtos funcionais (COOKE, CARNELL e WARDLE, 2006).

Constatou-se diferença significativa $(p<0,05)$ entre os escores obtidos para a aceitação do leite quando comparadas as sessões 2 e 3 (Tabela 2). O leite alcançou maior escore no teste com conhecimento sobre os benefícios do consumo da melatonina em ambos os grupos. Esses resultados mostram que os consumidores estão preocupados com a saúde e dispostos a aceitar produtos com características funcionais.

\section{TABELA 2 - COMPARAÇÃO ENTRE AS SESSÕES 2 E 3 USANDO O TESTE T PARA AMOSTRAS PAREADAS}

\begin{tabular}{cccc}
\hline \hline Voluntários & Sessão 2 & Sessão 3 & \\
& Escores médios & Escores médios & $\rho$-valor* \\
\cline { 2 - 4 } Menores de 50 anos & 5,6 & 5,9 & $<0,0001$ \\
Maiores de 50 anos & 5,6 & 6,5 & $<0,0001$ \\
\hline \hline
\end{tabular}

${ }^{*} \rho$-valor obtido no teste t para amostras pareadas.

Vidigal et al. (2011) observaram atitudes positivas dos consumidores em relação à funcionalidade dos produtos. Verificaram aumento nos escores de aceitação de sucos de frutas exóticas, quando acompanhadas de informações sobre seus benefícios à saúde. Segundo Siro et al. (2008), os produtos funcionais representam nova classe de alimentos com mercado em constante crescimento, porém seu desenvolvimento representa grande desafio - produzir alimentos saudáveis que agradem os consumidores quanto às características sensoriais e não sensoriais.

Ainformação de benefício à saúde elevou os escores de aceitação do leite com concentração aumentada de melatonina, mostrando que estratégias de marketing usando a divulgação dessas informações são importantes para o sucesso do produto no mercado.

\subsection{COMPARAÇÃO ENTRE OS GRUPOS DE CONSUMIDORES (IDADE INFERIOR E SUPERIOR A 50 ANOS)}

O resultado da análise de variância $(p<0,05)$ indicou diferenças estatísticas significativas entre os grupos de consumidores somente na sessão 3, conforme a Figura 6.

Não houve diferença significativa $(p>0,05)$ entre os escores médios de aceitação do grupo com idade superior a 50 anos e do grupo com idade inferior a 50 anos na sessão 1. Para ambos os grupos foi constatado escore médio de aceitação de aproximadamente 5,1, variando entre os termos hedônicos "gostei" e "nem gostei/nem desgostei". Na sessão 2, também não houve diferença significativa $(p>0,05)$ entre os dois grupos, ou seja, a influência da informação da presença de melatonina causou impacto semelhante nos escores de aceitação dos dois grupos. Para o grupo com idade inferior a 50 anos, a média dos escores de aceitação aumentou para 5,6. Para o grupo de voluntários com idade superior a 50 anos, o escore médio da segunda sessão também alcançou 5,6. Em ambos os grupos houve influência positiva da informação da presença de melatonina, apesar de os participantes não conhecerem o composto.

Verificou-se diferença significativa entre os grupos na sessão 3, sendo os voluntários com idade superior a 50 anos mais influenciados pelo conhecimento dos benefícios do consumo da melatonina que o grupo com idade inferior a 50 anos. $O$ escore médio de aceitação do grupo mais jovem foi de 5,9, enquanto o do grupo mais idoso alcançou escore médio de 6,5. A explicação sobre 
a maior importância dada ao conhecimento a respeito dos benefícios da melatonina pelas pessoas com idade superior a 50 anos reside na grande incidência de insônia nesse grupo, causada pelo declínio na produção de melatonina. Trabalho desenvolvido por Orwoll (2005) mostrou que $44 \%$ das pessoas com idade superior a 64 anos apresentavam problemas para dormir. Justifica-se assim, o fato das pessoas com idade superior a 50 anos serem mais influenciadas pelo conhecimento do benefício do consumo do leite que ajuda a melhorar a qualidade do sono.
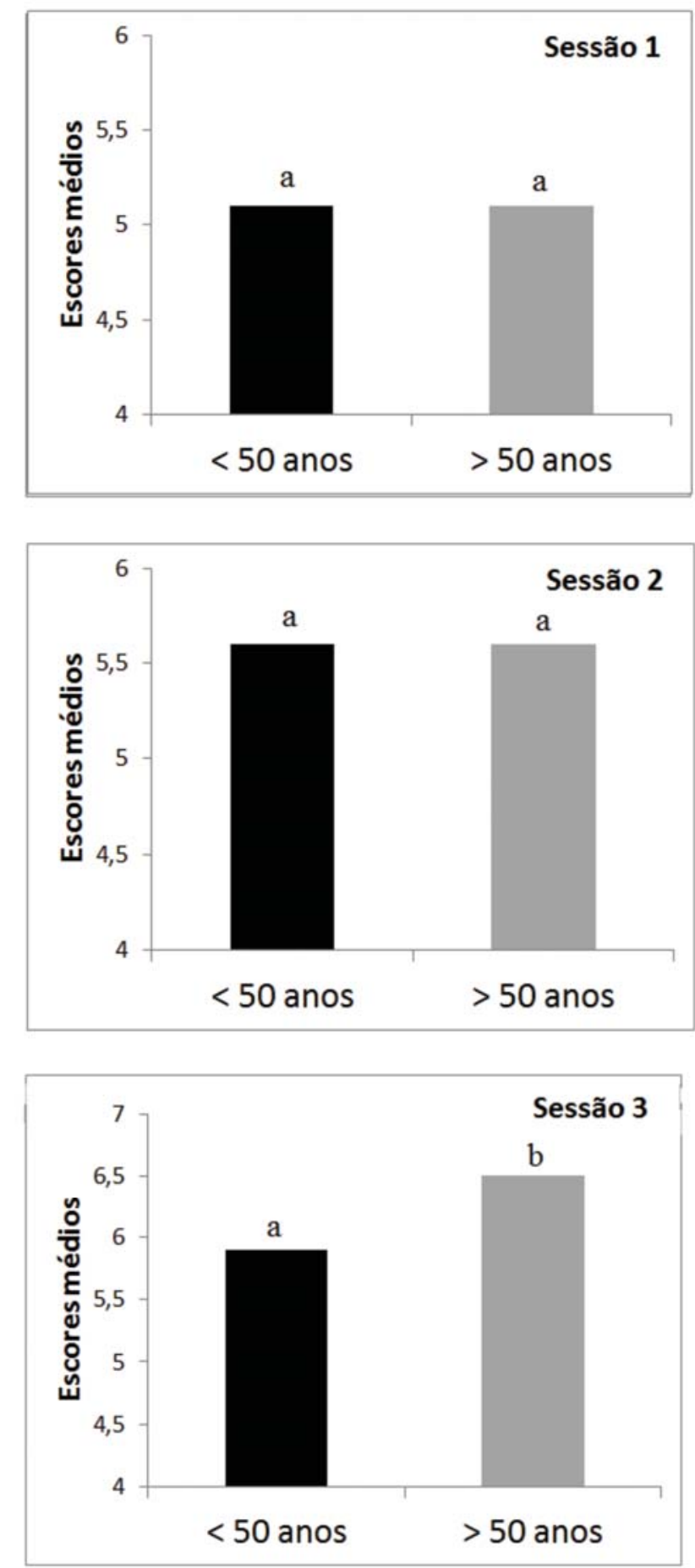

FIGURA 6 - MÉDIAS DOS ESCORES DE ACEITAÇÃO DO LEITE COM CONCENTRAÇÃO AUMENTADA DE MELATONINA, AVALIADOS NAS SESSÕES 1, 2 E 3

Médias com a mesma letra não diferem significativamente $(p<0,05)$ na mesma sessão. 


\section{CONCLUSÃO}

A informação da presença de melatonina e o conhecimento sobre seus benefícios à saúde elevaram os escores de aceitação do leite com concentração aumentada de melatonina, mostrando que estratégias de marketing usando a divulgação dessas informações são importantes para o sucesso do produto no mercado.

As pessoas com idade superior a 50 anos apresentaram maior interesse pelo leite com concentração aumentada de melatonina, sendo mais influenciadas pela informação sobre seus benefícios. Verificou-se também que a neofobia alimentar não influenciou a aceitação dos consumidores, pois a maioria ( $82 \%$ ) desconhecia tal composto. Mesmo assim, quando foi fornecida a informação de que o leite continha melatonina, os escores de aceitação do produto aumentaram. Assim, a palavra melatonina pode ser usada nas embalagens e propagandas desse leite, pois não foi detectada neofobia em relação ao termo.

\section{ABSTRACT \\ THE IMPACT OF INFORMATION ABOUT MELATONIN ON THE ACCEPTABILITY OF MILK}

This study aimed at evaluating the influence of information and knowledge upon the presence of melatonin in the acceptance of milk. Two hundred volunteers (aged under and over 50 years) were recruited to assess the pasteurized cow milk with increased concentration of melatonin. This study was separated in 3 stages: blind test, test with information about the presence of melatonin and testing of knowledge of the benefits of melatonin. All sessions were conducted in laboratory conditions, using 7-point hedonic scale. The results were analyzed by ANOVA and t test. The milk with information about the presence of melatonin had higher scores when compared to the blind test in both groups. By comparing the tests with and without knowledge on melatonin, it was noticed that information on its benefits has favored the product acceptability, mainly in the group aged over 50 years. It was therefore concluded that consumers are concerned about their health and willing to accept products with functional features.

KEY-WORDS: MELATONIN; ACCEPTABILITY AND INFLUENCE OF INFORMATION.

\section{REFERÊNCIAS}

1 ALLISON, A.M.A.; GUALTIERI, T.; CRAIG-PETSINGER, D. Are young teens influenced by increased product description detail and branding consumer testing? Food Quality and Preference, v.15, p.819-829, 2004.

2 BECH-LARSEN, T.; GRUNERT, K.G. The perceived healthiness of functional foods - a conjoint study of Danish, Finnish and American consumers' perception of functional foods. Appetite, v.40, p.9-14, 2003.

3 COOKE, L.; CARNELL, S.; WARDLE, J. Food neophobia and meal time food consumption in 4-5 year old children. International Journal of Behavioral Nutrition and Physical Activity, v.3, p.1-6, 2006.

4 DELLA LUCIA, S.M.; MINIM, V.P.R.; SILVA, C.H.O.; MINIM, L.A.; CERESINO, E.B. Expectativas geradas pela marca sobre a aceitabilidade de cerveja: estudo da interação entre características não sensoriais e o comportamento do consumidor. Boletim do CEPPA, v.28, n.1, p.11-24, jan./jun.2010.

5 HAILU, G.; BOECKER, A.; HENSON, S.; CRANFIELD, J. Consumer valuation of functional foods and neutraceuticals in Canada. A conjoint study using probiotics. Appetite, v.52, p.257-265, 2009.

6 JAEGER, S.R. Non-sensory factors in sensory science research. Food Quality and Preference, v.17, p.132-144, 2006.

7 KENNAWAY, D.J.; WRIGHT, H. Melatonin and circadian rhythms. Current Topics in Medicinal Chemistry, v.2, p.199209, 2002.

8 MINIM, V.P.R. Análise sensorial: estudo com consumidores. 2. ed. Viçosa, MG: Editora da Universidade Federal de Viçosa, 2010. $308 \mathrm{p}$

9 ORWOLL, M.M. Epidemiology of insomnia: what we know and what we still need to learn. Sleep Medicine Reviews, v.6, p.97-111, 2005. 
10 ÖZER, B.H.; KIRMACI, H. Functional milks and dairy beverages. International Journal of Dairy Technology, v.63, n.1, p.1-15, 2010.

11 PRESCOTT, J.; YOUNG, O.; O’NEILL, L.; YAU, N.J.N.; STEVENS, R. Motives for food choice: a comparison of consumers from Japan, Taiwan, Malaysia and New Zealand. Food Quality and Preference, v.13, p.489-495, 2002.

12 SABBE, S.; VERBEKE, W.; DELIZA, R.; VAN DAMME, P. Effect of a health claim and personal characteristics on consumer acceptance of fruit juices with different concentrations of açaí (Euterpe oleracea Mart.). Appetite, v.53, p.84-92, 2009.

13 SAS INSTITUTE INC. Statistical Analysis System. Version 9.1. Cary, NC, 2008.

14 SIEGRIST, M.; STAMPFLI, N.; KASTENKOLZ, H. Consumers' willingness to buy functional foods. The influence of carrier, benefit and trust. Appetite, v.51, p.526-529, 2008.

15 SIRO, I.; KAPOLNA, E.; KAPOLNA, B.; LUGASI, A. Functional food. Product development, marketing and consumer acceptance: a review. Appetite, v.51, p.456-467, 2008.

16 URALA, N.; LÄHTEENMÄKI, L. Attitudes behind consumers' willingness to use functional foods. Food Quality and Preference, v.15, p.793-803, 2004.

17 VAN TRIJP, H.C.M.; VAN DER LANS, I.A. Consumer perceptions of nutrition and health claims. Appetite, v.48, p.305-324, 2007.

18 VAN KLEEF, E.; VAN TRIJP, H.C.M.; LUNING, P. Functional foods: health claim food product compatibility and the impact of health claim framing on consumer evaluation. Appetite, v.44, p.299-308, 2005.

19 VERBEKE, W. Consumer acceptance of functional foods: socio-demographic, cognitive and attitudinal determinants. Food Quality and Preference, v.16, p.45-57, 2005.

20 VERBEKE, W. Functional foods: consumer willingness to compromise on taste for health? Food Quality and Preference, v.17, p.126-131, 2006.

21 VIDIGAL C.T.R.; MINIM, V.P.R.; CARVALHO, B.N.; MILAGRES, M.P.; GONÇALVES, A.C.A. Effect of a health claim on consumer acceptance of exotic Brazilian fruit juices: açaí (Euterpe oleracea Mart.), camu-camu (Myrciaria dubia), cajá (Spondias Lutea L.) and umbu (Spondias tuberosa Arruda). Food Research International, v.44, p.1988-1996, 2011.

\section{AGRADECIMENTOS}

À Coordenação de Aperfeiçoamento de Pessoal de Nível Superior (CAPES) e à Fundação de Amparo a Pesquisa de Minas Gerais (FAPEMIG) pelo apoio financeiro, e ao Departamento de Ciência e Tecnologia de Alimentos da Universidade Federal de Viçosa (UFV) pelo desenvolvimento da pesquisa. 